\title{
The clinical significance of reduced programmed cell death 5 expression in human gastrointestinal stromal tumors
}

\author{
FEI GAO ${ }^{1}$, LINGLING DING $^{2}$, MIAOQING ZHAO ${ }^{3}$, ZHONGHUA QU $^{1}$, \\ SHANYING HUANG ${ }^{1}$ and LINING ZHANG ${ }^{4}$ \\ ${ }^{1}$ Key Laboratory of Cardiovascular Remodeling and Function Research, Chinese Ministry of Education and \\ Chinese Ministry of Health, Shandong University Qilu Hospital; ${ }^{2}$ Center for Reproductive Medicine, \\ ${ }^{3}$ Department of Pathology, Shandong Provincial Hospital, ${ }^{4}$ Institute of Immunology, \\ School of Medicine, Shandong University, Jinan, Shandong, P.R. China
}

Received July 2, 2012; Accepted August 23, 2012

DOI: $10.3892 /$ or.2012.2023

\begin{abstract}
Programmed cell death 5 (PDCD5) is a novel apoptosis-related gene with potent antitumor effects which can interact with the histone acetyltransferase Tip60 and induce DNA damage-induced apoptosis. Reduced PDCD5 expression has been found in a few types of human tumors and is also associated with the progression and prognosis of the tumors. However, the expression status and clinical significance of PDCD5 in gastrointestinal stromal tumors has not yet been analyzed. In our study, we examined PDCD5 expression in 63 tumor samples of gastrointestinal stroma at both mRNA and protein levels by RT-PCR, western blotting and immunohistochemistry. We found that $57 \%(16 / 28)$ of the tumor samples had a decreased PDCD5 expression at the mRNA level and $53 \%(35 / 66)$ of the samples were found to have decreased PDCD5 expression at the protein level, whereas PDCD5 was highly expressed in all adjacent normal gastrointestinal tissues at the mRNA or protein level. Moreover, decreased PDCD5 expression was significantly associated with clinicopathological characteristics including tumor size and mitosis. Our results suggest that PDCD5 expression plays a significant role in the malignant progression of human gastrointestinal stromal tumors and may be a key inhibitory factor.
\end{abstract}

\section{Introduction}

Programmed cell death 5 (PDCD5) is a novel apoptosisrelated gene which was cloned from the human leukemia cell line TF-1 and designated TFAR19 (TF1 cell apoptosis-related

Correspondence to: Dr Fei Gao, Key Laboratory of Cardiovascular Remodeling and Function Research, Chinese Ministry of Education and Chinese Ministry of Health, Shandong University Qilu Hospital, Wen Hua Xi Road, Jinan 250012, Shandong, P.R. China

E-mail: gaofeijn@yahoo.com.cn

Key words: programmed cell death 5, expression, gastrointestinal stromal tumor, clinical significance gene 19) (1). It was able to induce DNA damage-induced apoptosis through interacting with the histone acetyltransferase Tip60 and phosphorylated in vitro or in vivo by the multifunctional kinase CK2 (2,3). The introduction of anti-TFAR19 antibody into HeLa cells could suppress apoptosis (4). In addition, PDCD5 fragments could suppress the tumorigenesis by inhibiting the Ras/Raf/MEK/ERK signaling pathway (5). Recent studies have also revealed that decreased PDCD5 expression has been observed in multiple types of human tumor cells and cancer including ovarian carcinomas (6), high-grade astrocytic gliomas (7), chondrosarcoma (8), colorectal cancer (9), myeloma (10), prostate cancer (11), acute myeloid leukemia (12), renal clear cell carcinoma (13), bladder carcinoma (14) and lung cancer (15). Decreased PDCD5 expression also correlates with tumor progression and prognosis. It has been demonstrated that PDCD5 expression in the gastric tumor tissues was significantly lower than that of the normal tissues and it improved the prognosis of patients compared with that of decreased PDCD5 expression (16). These findings suggest that PDCD5 might represent a novel tumor suppressor gene.

Gastrointestinal stromal tumors (GISTs) are non-epithelial, mesenchymal tumors, $30 \%$ of which are malignant or have a high potential for malignancy, although it comprises $<1 \%$ of all gastrointestinal tumors (17). The 5-year survival rate following surgery of GISTs is approximately 50-65\% (18). The overall survival of the patients suffering from the disease has improved slightly, although the treatment-resistant tumors have a well-recognized, well-understood, and treatable tumor entity within only one decade (19). There have been marked achievements in finding molecular targeted agents that cause the progression and metastasis of GISTs (20). It is now known that approximately $85 \%$ of GISTs arise from the mutations in KIT or the homologous receptor tyrosine kinase plateletderived growth factor receptor alpha (PDGFRA) gene (19). Therefore, molecular biological studies of the features of the tumor are key in understanding the mechanisms underlying the pathogenesis of GISTs, and, in turn, providing insights into therapeutics.

To our knowledge, there have been no studies on the clinical significance of PDCD5 expression in GISTs. In the present study, we investigated the PDCD5 expression levels by 

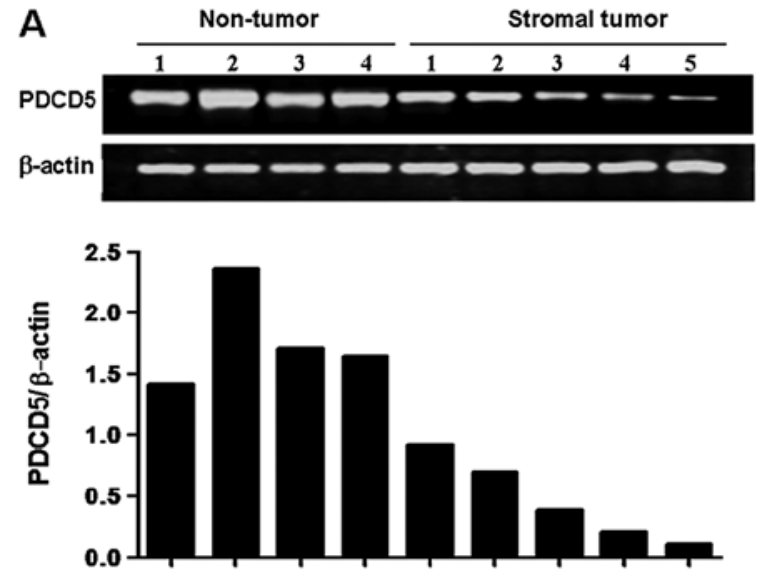

B

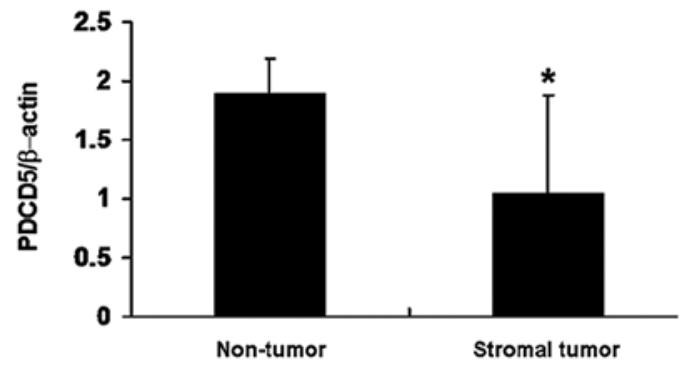

Figure 1. Expression of PDCD5 mRNA in frozen samples of gastrointestinal stromal tumors detected by RT-PCR. (A) The expression levels of PDCD5 mRNA in human non-tumor gastrointestinal tissues and in primary gastrointestinal stromal tumors were detected by RT-PCR. Data were normalized to $\beta$-actin. (B) Normalized PDCD5 mRNA levels in 13 non-tumor gastrointestinal tissues and 28 GIST samples were analyzed. The difference is statistically significant $\left({ }^{*} \mathrm{P}<0.05\right)$.

RT-PCR, western blotting and immunohistochemistry (IHC) in GISTs. We examined lower PDCD5 expression in the tumors compared with that of adjacent normal gastrointestinal tissues. In addition, we found that decreased PDCD5 expression was associated with the clinical pathological characteristics of GISTs.

\section{Materials and methods}

Tumor samples. Sixty-six GIST specimens (28 frozen and 38 paraffin-embedded tissues) were obtained from patients aged between 40 and 70 years (median, 60 years), who underwent surgery at the Department of General Surgery, Qilu Hospital and Shandong Provincial Hospital, Shandong University, from 2006 to 2010. None of the patients had received adjuvant immunosuppressive treatments including chemotherapy or radiotherapy prior to the surgery in order to eliminate their effects on gene expression. Tumor specimens were immediately frozen in liquid nitrogen following surgery. The normal non-tumor tissues were obtained from the adjacent area of the primary tumor. The study was in compliance with the ethics guidelines of the hospital, and all tumor samples were obtained with the patients' informed consent after the surgery. The analysis of clinicopathological tumor characteristics was performed according to the criteria of the National Institutes of Health (NIH) (21).

RNA isolation and semi-quantitative RT-PCR. Total-RNAs were extracted as previously described by the modified TRIzol $^{\circledR}$ one-step method and treated with DNase $(22,23)$. Total-RNAs $(3 \mu \mathrm{g})$ were subjected to reverse transcription to cDNA with the a kit. First-strand cDNA was synthesized using the Reverse-Transcribe kit (Promega, Madison, WI, USA) and PCR was performed using the gene-specific primers: sense 5'-CCATGG CGG ACG AGG AGC TTG-3', and anti-sense 5'-TCAATA ATC GTC ATC TTC ATC-3' for 30 cycles at $94^{\circ} \mathrm{C}$ for $30 \mathrm{sec}, 58^{\circ} \mathrm{C}$ for $30 \mathrm{sec}$ and $72^{\circ} \mathrm{C}$ for $30 \mathrm{sec}$ followed by an extension cycle at $72^{\circ} \mathrm{C}$ for $7 \mathrm{~min}$. Human $\beta$-actin primer was used as a positive control. Water instead of the cDNA template was used in negative control reactions. All PCR products were analyzed with $2 \%$ agarose gel electrophoresis. RT-PCR was performed at least three times for each sample.

SDS-PAGE and western blotting. The proteins were extracted from non-tumor and tumor samples using a modified TRIzol one-step extraction method. The concentration of the protein was determined by Bradford analysis (Bio-Rad, Hercules, CA, USA). The protein extract was dissolved in a loading buffer (1 mM Tris-Cl, 3\% SDS, 60\% glycerol and $75 \mathrm{mM}$ DTT) and each sample was separated and analyzed by SDS-PAGE on a $15 \%$ gel and transferred onto PVDF membranes. The PVDF membrane was incubated with anti-human PDCD5 or rabbit anti-human $\beta$-actin at $4{ }^{\circ} \mathrm{C}$ overnight. Immunoreactive bands were visualized using the enhanced chemiluminescence method according to the manufacturer's instructions (ECL, Amersham Biosciences, Little Chalfont, UK). Western blotting was performed at least three times for each sample.

Immunohistochemistry. Formalin-fixed, paraffin-embedded tissue sections from 28 fresh and 38 paraffin-embedded tissues were cut at 4-6 $\mu \mathrm{m}$ and transferred to slides. Tumor tissues were deparaffinized in xylene and rehydrated through alcohol gradient. The slides were washed, blocked for endogenous peroxidase activity, pre-incubated with goat serum, and were then stained with anti-PDCD5 polyclonal antibody overnight at $4^{\circ} \mathrm{C}$. Subsequently, the slides were incubated with biotin-conjugated anti-rabbit IgG and developed with an HRP-streptavidin-based ABC kit and a DAB Peroxidase Substrate kit (Maixin Co., Fuzhou, China). The nuclei were counterstained with hematoxylin. Negative controls for the specificity of IHC were carried out by replacing the primary antibody with non-immune rabbit IgG. The PDCD5 staining was divided into five (0-5) grades according to staining intensity: - (score 0$),+($ score 1$),++($ score 2$),+++($ score 3$),++++$ (score 4) and +++++ (score 5). The expression of PDCD5 was defined as follows: scores 0-3 were classified as weak expression of PDCD5, whereas 4 and 5 were graded as high expression of PDCD5. Slides were analyzed by two independent pathologists blind to tumor sample origin. All staining experiments were performed in duplicate for each sample. 
A
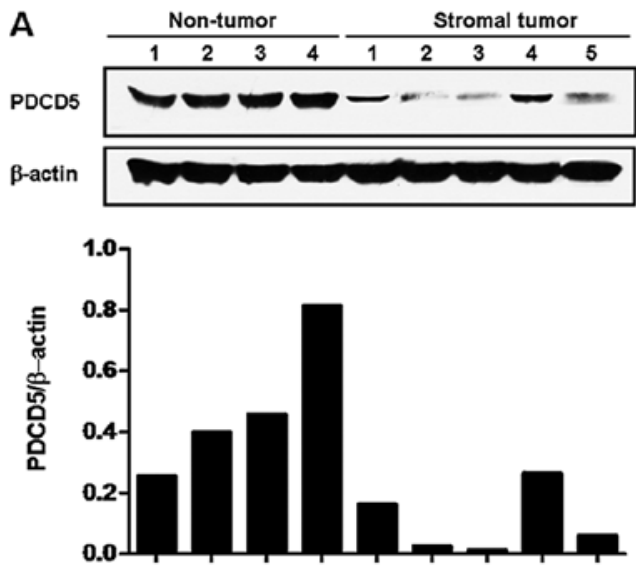

B

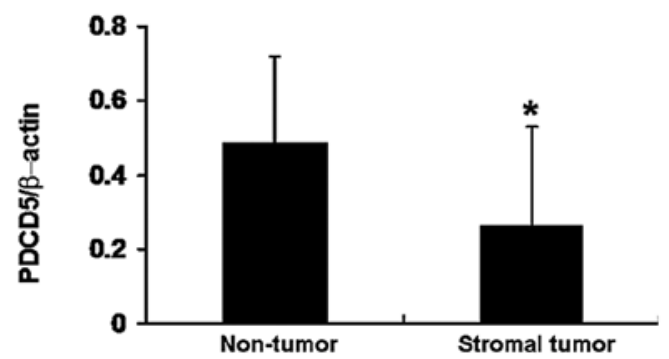

Figure 2. Expression of PDCD5 protein in gastrointestinal stromal tumors detected by western blotting. (A) The expression levels of PDCD5 protein in human non-tumor gastrointestinal tissues and in primary gastrointestinal stromal tumors were detected by western blotting. Data were normalized to $\beta$-actin. (B) Normalized PDCD5 protein levels in 13 non-tumor gastrointestinal tissues and 28 GIST samples were analyzed. The difference is statistically significant $($ P $<0.05)$.
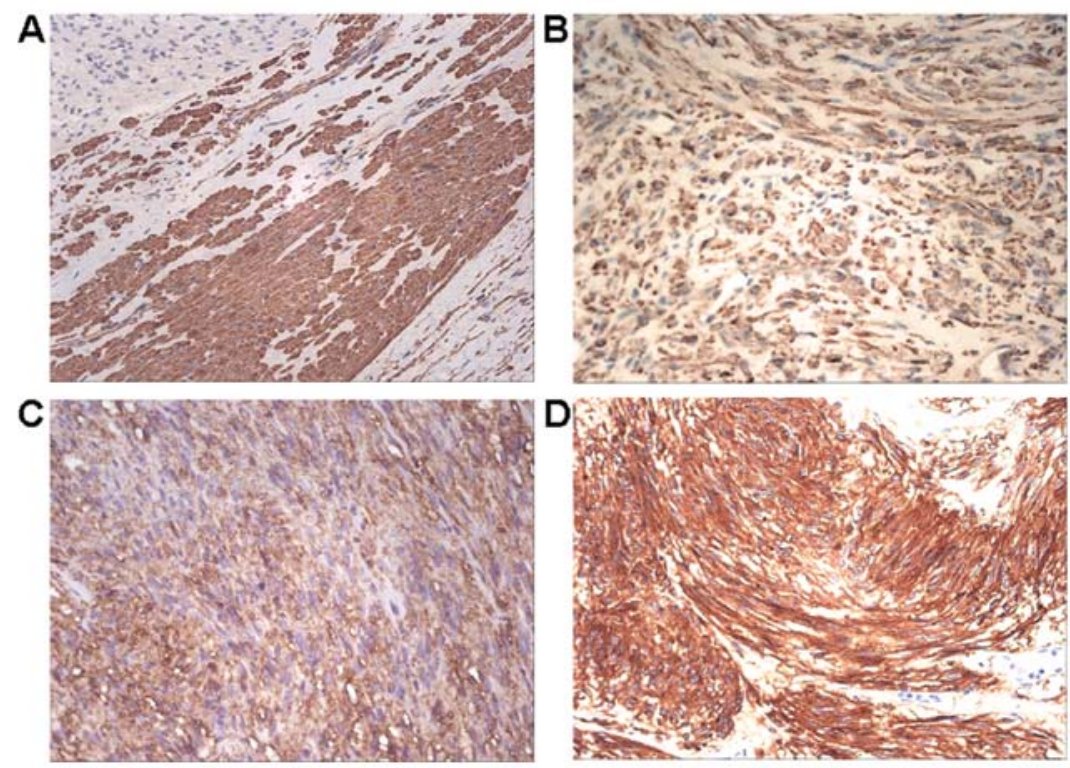

Figure 3. PDCD5 protein expression in gastrointestinal stromal tumors detected by immunohistochemistry. (A) Strong PDCD5 staining in non-tumor gastrointestinal tissues. (B-D) Decreased PDCD5 staining in gastrointestinal stromal tumors. (B) Score 1, (C) score 2, (D) score 4 (magnification, x400), as described in Materials and methods.

Statistical analysis. The $\chi^{2}$ test was used to analyze the correlation of expression of PDCD5 protein with clinicopathological parameters. Pearson's coefficient test was used to analyze the correlation between PDCD5 expression labeling index. $\mathrm{P}<0.05$ was considered to indicate statistically significant differences. All calculations were performed using the SPSS statistical software package.

\section{Results}

Expression of PDCD5 $\mathrm{mRNA}$ in GISTs. To explore the potential role of PDCD5 expression in primary GISTs, we first detected the expression of PDCD5 mRNA by semi-quantitative RT-PCR. As shown in Fig. 1A, non-tumorous tissues expressed high levels of PDCD5 mRNA, whereas 16/28 (57\%) of GIST samples showed low levels of PDCD5 mRNA expres- sion. These results suggested that the expression of PDCD5 at the mRNA levels was decreased in human GISTs compared with that in non-tumorous tissues (Fig. 1B).

Decreased expression of PDCD5 protein in GISTs. We further detected the expression of PDCD5 protein by western blotting and IHC. Compared with normal tissues adjacent to the tumor, the results of western blotting showed that PDCD5 protein expression in frozen GIST specimens was significantly decreased (Fig. 2). Additionally, we examined the expression of PDCD5 proteins by IHC. In non-tumorous tissues, there was a strong positive staining of PDCD5 (Fig. 3A). By contrast, PDCD5 expression of 28 frozen cases and 38 paraffinembedded sample tissues of GISTs exhibited weak or faint staining of PDCD5 protein (Fig. 3B-D). Among them, 53\% $(35 / 66)$ of tumor tissues expressed decreased expression of 


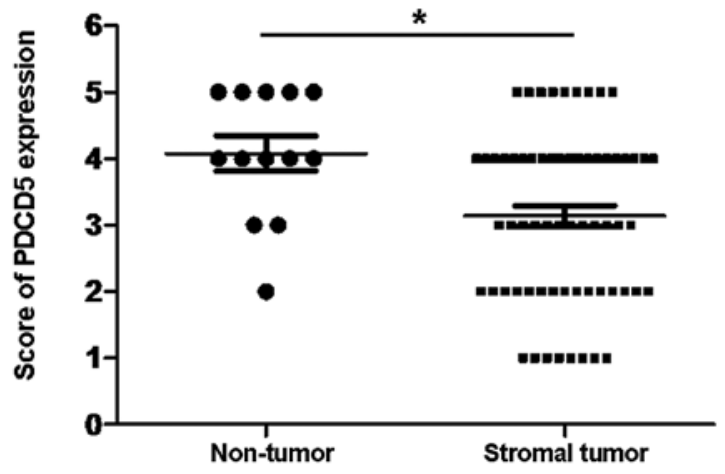

Figure 4. Distribution of PDCD5 staining in non-tumor gastrointestinal tissues and gastrointestinal stromal tumors. Overall level of PDCD5 expression was significantly lower in gastrointestinal stromal tumors compared with non-tumor gastrointestinal tissues according to the results of immunohistochemistry $\left({ }^{*} \mathrm{P}<0.05\right)$.

Table I. PDCD5 expression in GISTs.

\begin{tabular}{lccc}
\hline Parameters & PDCD5 & PDCD5 & P-value \\
& Low expression & High expression & \\
\hline
\end{tabular}

\begin{tabular}{lrrr}
\hline $\begin{array}{l}\text { Total no. of } \\
\text { patients }\end{array}$ & 35 & 31 & \\
Gender & & & \\
Male & 19 & 17 & 0.964 \\
Female & 16 & 14 & \\
Age (years) & & & \\
$\geq 60$ & 21 & 16 & 0.493 \\
$<60$ & 14 & 15 & \\
Risk group & & & \\
High risk & 14 & 8 & \\
Non-high risk & 21 & 23 & \\
Tumor size & & & \\
$\leq 5 \mathrm{~cm}$ & 6 & 15 & \\
$5-10 \mathrm{~cm}$ & 23 & 12 & \\
$\geq 10 \mathrm{~cm}$ & 6 & 4 & \\
Mitosis & & & \\
$\leq 5 / 50$ HPF & 17 & 23 & \\
$5-10 / 50 \mathrm{HPF}$ & 8 & $643^{\mathrm{a}}$ \\
$\geq 10 / 50 \mathrm{HPF}$ & 10 & & \\
\hline
\end{tabular}

${ }^{a} \mathrm{P}<0.05$. PDCD5 expression in GISTs. The clinical parameters include gender, age, risk group, tumor size and mitosis.

PDCD5 protein by IHC analysis, and the overall expression of PDCD5 in GISTs was significantly lower compared with normal tissues adjacent to tumor (Fig. 4).

Correlation of PDCD5 expression at the protein level with the clinicopathological characteristics in primary GISTs. To determine the clinical value of decreased PDCD5 expression in primary GISTs, we further examined the correlation of
PDCD5 expression with the clinicopathological parameters, such as age, gender, tumor size, mitosis and risk group. The results showed no significant relationship between PDCD5 expression and age, gender and risk group. However, PDCD5 expression significantly correlated with tumor size $(\mathrm{P}<0.05)$ and mitosis $(\mathrm{P}<0.05)$ of patients (Table $\mathrm{I})$, suggesting that PDCD5 expression might be associated with the malignant progression of GISTs.

\section{Discussion}

Previous studies have confirmed that PDCD5 is a novel apoptosis-related gene, and research from mice and human tumors has shown that PDCD5 might be a new tumor suppressor gene that significantly affects tumor development and prognosis $(25,26)$. PDCD5 could inhibit the growth of tumor cells through the induction of apoptosis and the inhibition of the cell cycle $(27,28)$. Previously, we had also found that diminished PDCD5 expression existed in the high-grade astrocytic gliomas and epithelial ovarian carcinomas $(6,7)$. However, the clinical role of PDCD5 in human GISTs, including the relationship with tumor progression was, to date, unclear.

Gastrointestinal stromal tumors (GISTs) are the most common primary mesenchymal tumors of the gastrointestinal tract (24). It was distinguished from other similar tumors in which the presence of a KIT protein and the possibility of kit mutations was discovered. A large-scale clinical trial indicated that $85 \%$ of GISTs had an active mutation in the kit protooncogene, while only 3-5\% mutation of PDGFRA existed in GISTs (29). Although targeted agents, such as imatinib, were studied for the treatment of the tumor, $40-50 \%$ of patients developed imatinib resistance within the first two years of therapy. Most cases of primary resistance appeared kit and PDGFRA wild-type (30). With the advancement in understanding of the molecular biology, GIST progresses together with improvement in immunohistochemical staining. Many more target molecules are required to prevent the high incidence of recurrence.

In the present study, we demonstrated for the first time, that PDCD5 expression was decreased in human primary GISTs at the mRNA or protein levels. Statistical analysis showed that the overall expression level of PDCD5 in tumor samples was markedly reduced compared with adjacent non-tumor normal tissues. To date, little is known about the regulation mechanisms of abnormal PDCD5 expression. Downregulation of PDCD5 expression was found in human breast epithelial cells after bisphenol A exposure accompanied by DNA methylation. These suggested that the abnormal expression of PDCD5 might be associated with DNA methylation and required further investigations in order to be determined. Therefore, further studies regarding the detailed molecular mechanisms of abnormal expression of the gene in GISTs are required.

In addition, we also discovered that the decreased expression of PDCD5 proteins was significantly associated with the tumor size and mitosis of GISTs. It has been reported that the reduced expression of PDCD5 correlates with short survival periods of patients with gastric tumor tissues (16). At the same time, we also found that lost or decreased PDCD5 expression in serous cystadenocarcinomas was associated significantly with FIGO stage and poorer disease-specific survival of 
patients (6). These suggested that reduced PDCD5 expression might contribute to the pathogenesis of human GISTs and PDCD5 might be an important suppressor gene impacting the malignant progression of the disease.

In conclusion, results of the present study show that PDCD5 might serve as a novel therapeutic target for the treatment of the tumor and the downregulation of PDCD5 gene expression might be a key factor in the malignant progression of GISTs. However, the detailed regulation mechanism of decreased PDCD5 expression in GISTs requires further investigation.

\section{Acknowledgements}

This study was supported by the National 973 Basic Research Program of China (no. 2012CB722406), the National Natural Science Foundation of China (no. 81000126), the Natural Science Foundation of Shandong Province (no.ZR2009CM0212009ZRB01192) and Grants from the Specialized Research Fund for the Doctoral Program of Higher Education of China (no. 20090131120066).

\section{References}

1. Liu H, Wang Y, Zhang Y, et al: TFAR19, a novel apoptosisrelated gene cloned from human leukemia cell line TF-1, could enhance apoptosis of some tumor cells induced by growth factor withdrawal. Biochem Biophys Res Commun 254: 203-210, 1999.

2. Xu L, Chen Y, Song Q, Xu D, Wang Y and Ma D: PDCD5 interacts with Tip60 and functions as a cooperator in acetyltransferase activity and DNA damage-induced apoptosis. Neoplasia 11: 345-354, 2009

3. Salvi M, Xu D, Chen Y, Cabrelle A, Sarno S and Pinna LA: Programmed cell death protein 5 (PDCD5) is phosphorylated by CK2 in vitro and in 293T cells. Biochem Biophys Res Commun 387: 606-610, 2009.

4. Rui M, Chen Y, Zhang Y and Ma D: Transfer of anti-TFAR19 monoclonal antibody into HeLa cells by in situ electroporation can inhibit the apoptosis. Life Sci 71: 1771-1778, 2002.

5. Han XR, Sun Y and Bai XZ: The anti-tumor role and mechanism of integrated and truncated PDCD5 proteins in osteosarcoma cells. Cell Signal 24: 1713-1721, 2012.

6. Zhang X, Wang X, Song X, et al: Clinical and prognostic significance of lost or decreased PDCD5 expression in human epithelial ovarian carcinomas. Oncol Rep 25: 353-358, 2011.

7. Li H, Wang Q, Gao F, et al: Reduced expression of PDCD5 is associated with high-grade astrocytic gliomas. Oncol Rep 20: 573-579, 2008.

8. Chen $\mathrm{C}$, Zhou H, Xu L, et al: Prognostic significance of downregulated expression of programmed cell death 5 in chondrosarcoma. J Surg Oncol 102: 838-843, 2010.

9. Yin A, Jiang Y, Zhang X, Zhao J and Luo H: Transfection of PDCD5 sensitizes colorectal cancer cells to cisplatin-induced apoptosis in vitro and in vivo. Eur J Pharmacol 649: 120-126, 2010.

10. Bao L, Ruan GR, Lu XJ, et al: Abnormal expression of programmed cell death 5 gene in multiple myeloma patients. Zhongguo Shi Yan Xue Ye Xue Za Zhi 18: 634-637, 2010 (In Chinese)

11. Du YJ, Xiong L, Lou Y, Tan WL and Zheng SB: Reduced expression of programmed cell death 5 protein in tissue of human prostate cancer. Chin Med Sci J 24: 241-245, 2009.
12. Ruan GR, Chen SS, Ma X, et al: Abnormal expression of PDCD5 in the bone marrow cells of adult acute myeloid leukemia. Zhongguo Shi Yan Xue Ye Xue Za Zhi 15: 462-465, 2007 (In Chinese).

13. Tan WL, Xiong L, Zheng SB, et al: Relationship between programmed cell death 5 protein expression and prognosis of renal clear cell carcinoma. Nan Fang Yi Ke Da Xue Xue Bao 26: 1316-1318, 2006 (In Chinese).

14. Xiong L, Tan WL, Yu ZC, et al: Expression of TFAR19 (PDCD5) in normal human kidney, renal clear cell carcinoma, normal human bladder and bladder carcinoma. Nan Fang Yi Ke Da Xue Xue Bao 26: 805-809, 2006 (In Chinese).

15. Spinola M, Meyer P, Kammerer S, et al: Association of the PDCD5 locus with lung cancer risk and prognosis in smokers. J Clin Oncol 24: 1672-1678, 2006.

16. Yang YH,Zhao M, Li WM, et al: Expression of programmed cell death 5 gene involves in regulation of apoptosis in gastric tumor cells. Apoptosis 11: 993-1001, 2006.

17. Paral J, Slaninka I, Kalabova H and Hadzi-Nikolov D: Gastrointestinal stromal tumors: review on morphology, molecular pathology, diagnostics, prognosis and treatment options. Acta Gastroenterol Belg 73: 349-359, 2010.

18. Lai Eric CH, Lau Stephanie HY and Lau WY: Current management of gastrointestinal stromal tumors - a comprehensive review. Int J Surg 10: 334-340, 2012.

19. Bayraktar UD, Bayraktar S and Rocha-Lima CM: Molecular basis and management of gastrointestinal stromal tumors. World J Gastroenterol 16: 2726-2734, 2010.

20. Liegl-Atzwanger B, Fletcher JA and Fletcher CD: Gastrointestinal stromal tumors. Virchows Arch 456: 111-127, 2010.

21. Fletcher CD, Berman JJ, Corless C, et al: Diagnosis of gastrointestinal stromal tumors: a consensus approach. Int J Surg Pathol 10: 81-89, 2002.

22. Chadderton T,Wilson C, Bewick M and Gluck S: Evaluation of three rapid RNA extraction reagents: relevance for use in RT-PCR's and measurement of low level gene expression in clinical samples. Cell Mol Biol 43: 1227-1234, 1997.

23. Culley DE, Kovacik WP Jr, Brockman FJ and Zhang W: Optimization of RNA isolation from the archaebacterium Methanosarcina barkeri and validation for oligonucleotide microarray analysis. J Microbiol Methods 67: 36-43, 2006.

24. Laurini JA and Carter JE: Gastrointestinal stromal tumors: a review of the literature. Arch Pathol Lab Med 134: 134-141, 2010.

25. Xu HY, Chen ZW, Pan YM, et al: Transfection of PDCD5 effect on the biological behavior of tumor cells and sensitized gastric cancer cells to cisplatin-induced apoptosis. Dig Dis Sci 57: 1847-1856, 2012

26. Wang Y, Chu H, Zhou L, et al: Correlation of PDCD5 and apoptosis in hair cells and spiral ganglion neurons of different age of C57BL/6J mice. J Huazhong Univ Sci Technolog Med Sci 32: 113-118, 2012.

27. Ruan GR, Zhao HS, Chang Y, et al: Adenovirus-mediated PDCD5 gene transfer sensitizes K562 cells to apoptosis induced by idarubicin in vitro and in vivo. Apoptosis 13: 641-648, 2008.

28. Wang YF, Song QS, Zhang YM, Ma DL, Wang Y and Ke XY: Sensitizing effect of recombinant human PDCD5 protein on chemotherapy of acute monocytic leukemia cell line U937 and its mechanism. Zhongguo Shi Yan Xue Ye Xue Za Zhi 18: 277-281, 2010 (In Chinese).

29. de Matteo RP, Ballman KV, Antonescu CR, et al: Adjuvant imatinib mesylate after resection of localised, primary gastrointestinal stromal tumour: a randomised, double-blind, placebo-controlled trial. Lancet 373: 1097-1104, 2009.

30. Gramza AW, Corless CL and Heinrich MC: Resistance to tyrosine kinase inhibitors in gastrointestinal stromal tumors. Clin Cancer Res 15: 7510-7518, 2009. 